\title{
Politique
}

Politique

\section{Jean-Pierre Deslauriers (sous la direction de), Les méthodes de la recherche qualitative, Sillery, Presses de l'Université du Québec, 1987, 153 p.}

\section{Lawrence Olivier}

Numéro 12, automne 1987

Mouvements et acteurs

URI : https://id.erudit.org/iderudit/040579ar

DOI : https://doi.org/10.7202/040579ar

Aller au sommaire du numéro

Éditeur(s)

Société québécoise de science politique

ISSN

0711-608X (imprimé)

1918-6584 (numérique)

Découvrir la revue

Citer ce compte rendu

Olivier, L. (1987). Compte rendu de [Jean-Pierre Deslauriers (sous la direction de), Les méthodes de la recherche qualitative, Sillery, Presses de l'Université du Québec, 1987, 153 p.] Politique, (12), 159-162. https://doi.org/10.7202/040579ar d'utilisation que vous pouvez consulter en ligne.

https://apropos.erudit.org/fr/usagers/politique-dutilisation/ 
Jean-Pierre Deslauriers (sous la direction de), Les méthodes de la recherche qualitative, Sillery, Presses de l'Université du Québec, $1987,153 \mathrm{p}$.

Les méthodes qualitatives sont à l'ordre du jour en sciences sociales. Sur le constat d'un "savoir en crise» ou sur celui d'un échec de la quantification du social s'annonce le retour en force de la recherche qualitative. Un retour en force qui augure un renouvellement méthodologique et/ou épistémologique de nos disciplines. C'est dans cet horizon épistémologico-théorique que s'inscrit l'ouvrage de Jean-Pierre Deslauriers Les méthodes de la recheche qualitative. En effet, il ne s'agit pas seulement d'une présentation des différentes méthodes qualitatives (récits de vie, analyse de contenu, recheche-action, etc.) mais s'exprime dans l'ensemble des contributions qu'il contient l'idée qu'elles transforment la manière même de faire la recherche, la pratique des sciences sociales. 
Sans remettre en cause le bien fondé d'une telle idée, il convient cependant d'interroger le discours qui l'énonce pour rendre compte de ce qui est réellement en jeu dans ce qui s'annonce et se présente comme un renouveau de nos disciplines. Pour ce faire, il s'agit moins de considérer le contenu du discours, qui vise souvent à légitimer une pratique, que ses conditions de possibilité, le régime d'existence des énoncés en étudiant la manière dont il problématise la réalité qu'il cherche à imposer.

Le discours sur les méthodes de la recherche qualitative a pour condition la détermination d'une place, d'un lieu à partir duquel s'énonce le discours de vérité sur la réalité sociale. Il est représenté par une figure: l'intellectuel. Le constat d'échec de la sociologie «officielle» a un double objectif. Il vise à problématiser 1) la réalité sociale qu'étudient les sciences sociales; 2 ) à redéfinir le rôle et le statut du chercheur.

La «redécouverte» de l'acteur, de cette réalité occultée par les sociologies du système, de la structure, etc., permet de problématiser le réel. La réalité sociale ne se réduit pas aux symboles mathématiques ni aux relations entre les éléments d'un système. Elle est le produit de la dynamique des forces sociales, de la créativité des pratiques des acteurs sociaux (p. 14). D'où l'importance d'étudier «les phénomènes sociaux dans leur contexte ordinaire, habituel pour ne pas dire naturel» (p. 147). Et, c'est précisément parce qu'elle est organisée, structurée par l'activité des acteurs sociaux qu'il n'est plus possible d'utiliser les méthodes de l'objectivation scientifique pour appréhender la réalité sociale. Accepter l'importance de l'acteur, c'est remettre en cause nos critères de scientificité pour essayer d'y déployer un autre regard. Il suppose de nouvelles démarches de recherche qui se définissent par le rôle et la fonction qu'elles accordent au chercheur.

Le discours sur la recherche qualitative opère à ce niveau un déplacement épistémologique. Le chercheur ne peut plus se 
mettre à distance pour observer (objectivement) le réel. L'étude des pratiques sociales implique qu'il puisse comprendre les expériences des acteurs sociaux. Une saisie qui suppose un engagement. Le terme a ici une double acception. Il désigne d'abord l'échange entre acteurs et chercheurs. On parle d'enquête conjointe où l'acteur participe au processus de production de la connaissance. Il n'est plus considéré seulement comme un objet passif mais plutôt comme un sujet dynamique qui contribue, en racontant, par exemple, ses expériences vécues, à l'avancement de la recherche et de la connaissance. En retour, le chercheur s'implique dans «l'expérience» qu'il analyse. L'engagement signifie maintenant: intervention. En s'engageant, le chercheur participe aux projets sociaux des acteurs sociaux. Il, écrit Charbonneau, "chemine avec eux sur la route de leur changement, selon leurs perceptions leurs objectifs, leurs choix de moyens et leur rythme, faute de quoi on les manipule bassement par le «haut savoir' interposé» (p. 88). Le chercheur participe par son engagement à un processus dynamique de transformation sociale par les alliances qu'il noue avec les forces sociales. Le sujet épistémique des méthodes de la recherche qualitative refuse la séparation théorie/pratique pour une théorie de l'engagement où le savoir émane et est utilisé par les acteurs sociaux en fonction de leurs objectifs, de leur propre pratique; "politique». Il prend le statut d'intellectuel.

Depuis deux siècles, le statut du chercheur est redéfini et à travers lui l'usage que l'on peut et doit faire de la connaissance. La question a été de savoir s'il était possible en renonçant à l'objectivation et à la neutralité axiologique de garder aux sciences sociales le statut de scientificité. Débat stérile qui n’a guère renouvellé nos manières de connaître puisque le problème est moins d'affranchir le savoir du pouvoir que de se libérer de l'illusion de la vérité, du pouvoir de faire croire en montrant. La recherche qualitative non seulement n'échappe pas au pouvoir de la vérité 
mais elle la redouble d'une autre plus grande encore. Elle nous fait croire qu'il y va, dans la vérité, de notre libération et que le chercheur peut et doit y participer. Que l'acteur social en soit l'origine ou non c'est toujours au nom d'une représentation de la réalité que se justifie et se légitime la nécessité de la changer ou de la transformer. La croyance dans la vérité gouverne nos pratiques. Il me semble que si l'on veut changer nos manières d'appréhender la réalité, il faut d'abord interroger le pouvoir de faire croire de la vérité, c'est-à-dire les discours qui problématisent le réel et les théologiens qui les tiennent. La difficulté ce n'est pas d'utiliser d'une autre manière la connaissance mais de la concevoir comme «indifférente» et sans maître. Les méthodes de la recherche qualitative n'y contribue d'aucune manière. L'ouvrage de Deslauriers se limite à des prescriptions méthodologiques sur la manière de dire vrai.

Lawrence Olivier

Université du Québec à Montréal. 